\title{
Reversed version of a generalized Aczél's inequality and its application
}

Jing-Feng Tian*

\section{${ }^{*}$ Correspondence: \\ tianjfhxm_ncepu@yahoo.cn \\ College of Science and Technology, \\ North China Electric Power \\ University, Baoding, Hebei Province \\ 071051, P.R. China}

\begin{abstract}
In this paper, we give a reversed version of a generalized Aczél's inequality which is due to Wu and Debnath. As an application, an integral type of the reversed version of the Aczél-Vasić-Pečarić inequality is obtained.

MSC: Primary 26D15; secondary 26D10

Keywords: Aczél's inequality; Aczél-Vasić-Pečarić inequality; reversed version; generalization
\end{abstract}

\section{Introduction}

In 1956, Aczél [1] established the following inequality which is of wide application.

Theorem A If $a_{i}, b_{i}(i=1,2, \ldots, n)$ are positive numbers such that $a_{1}^{2}-\sum_{i=2}^{n} a_{i}^{2}>0$ or $b_{1}^{2}-$ $\sum_{i=2}^{n} b_{i}^{2}>0$, then

$$
\left(a_{1}^{2}-\sum_{i=2}^{n} a_{i}^{2}\right)\left(b_{1}^{2}-\sum_{i=2}^{n} b_{i}^{2}\right) \leq\left(a_{1} b_{1}-\sum_{i=2}^{n} a_{i} b_{i}\right)^{2} .
$$

It is well known that Aczél's inequality (1) plays an important role in the theory of functional equations in non-Euclidean geometry. Various refinements, generalizations and applications of inequality (1) have appeared in literature (see, e.g., [2-12], [13] and the references therein).

One of the most important results in the works mentioned above is the exponential generalization of (1) asserted by Theorem B.

Theorem B Let $p$ and $q$ be real numbers such that $p, q \neq 0$ and $\frac{1}{p}+\frac{1}{q}=1$, and let $a_{i}, b_{i}$ $(i=1,2, \ldots, n)$ be positive numbers such that $a_{1}^{p}-\sum_{i=2}^{n} a_{i}^{p}>0$ and $b_{1}^{q}-\sum_{i=2}^{n} b_{i}^{q}>0$. Then, for $p>1$, we have

$$
\left(a_{1}^{p}-\sum_{i=2}^{n} a_{i}^{p}\right)^{\frac{1}{p}}\left(b_{1}^{q}-\sum_{i=2}^{n} b_{i}^{q}\right)^{\frac{1}{q}} \leq a_{1} b_{1}-\sum_{i=2}^{n} a_{i} b_{i} .
$$

If $p<1(p \neq 0)$, we have the reverse inequality.

Remark 1.1 The case $p>1$ of Theorem B was proved by Popoviciu [8]. The case $p<1$ was given in [10] by Vasić and Pečarić.

(c) 2012 Tian; licensee Springer. This is an Open Access article distributed under the terms of the Creative Commons Attribution License (http://creativecommons.org/licenses/by/2.0), which permits unrestricted use, distribution, and reproduction in any medium, provided the original work is properly cited. 
In another paper [11], Vasić and Pečarić presented the following extension of inequality (1).

Theorem C Let $a_{r j}>0, \lambda_{j}>0, a_{1 j}^{\lambda_{j}}-\sum_{r=2}^{n} a_{r j}^{\lambda_{j}}>0, r=1,2, \ldots, n, j=1,2, \ldots, m$, and let $\sum_{j=1}^{m} \frac{1}{\lambda_{j}} \geq 1$. Then

$$
\prod_{j=1}^{m}\left(a_{1 j}^{\lambda_{j}}-\sum_{r=2}^{n} a_{r j}^{\lambda_{j}}\right)^{\frac{1}{\lambda_{j}}} \leq \prod_{j=1}^{m} a_{1 j}-\sum_{r=2}^{n} \prod_{j=1}^{m} a_{r j} .
$$

Recently, it comes to our attention that an interesting generalization of Aczél's inequality, which was established by Wu and Debnath in [14], is as follows.

Theorem D Let $a_{r j}>0, \lambda_{j}>0, a_{1 j}^{\lambda_{j}}-\sum_{r=2}^{n} a_{r j}^{\lambda_{j}}>0, r=1,2, \ldots, n, j=1,2, \ldots, m$, and let $\rho=$ $\min \left\{\sum_{j=1}^{m} \frac{1}{\lambda_{j}}, 1\right\}$. Then

$$
\prod_{j=1}^{m}\left(a_{1 j}^{\lambda_{j}}-\sum_{r=2}^{n} a_{r j}^{\lambda_{j}}\right)^{\frac{1}{\lambda_{j}}} \leq n^{1-\rho} \prod_{j=1}^{m} a_{1 j}-\sum_{r=2}^{n} \prod_{j=1}^{m} a_{r j}
$$

and equality holds if and only if $a_{1 j}=n^{\frac{1}{p_{j}}} a_{2 j}=\cdots=n^{\frac{1}{p_{j}}} a_{n j}, j=1,2, \ldots, m$ for $\rho<1$, or

$$
\frac{a_{11}^{\lambda_{1}}}{a_{1 j}^{\lambda_{j}}}=\frac{a_{21}^{\lambda_{1}}}{a_{2 j}^{\lambda_{j}}}=\cdots=\frac{a_{n 1}^{\lambda_{1}}}{a_{n j}^{\lambda_{j}}}, \quad j=2,3, \ldots, \text { m for } \rho=1 .
$$

The purpose of this work is to give a reversed version of inequality (4). As application, an integral type of the reversed version of the Aczél-Vasić-Pečarić inequality is obtained.

\section{Reversed version of a generalized Aczél's inequality}

We need the following lemmas in our deduction.

Lemma 2.1 [5] If $x_{i} \geq 0, \lambda_{i}>0, i=1,2, \ldots, n, 0<p \leq 1$, then

$$
\sum_{i=1}^{n} \lambda_{i} x_{i}^{p} \leq\left(\sum_{i=1}^{n} \lambda_{i}\right)^{1-p}\left(\sum_{i=1}^{n} \lambda_{i} x_{i}\right)^{p} .
$$

The inequality is reversed for $p \geq 1$ or $p<0$. In each case, the sign of the equality holds if and only if $x_{i}=x_{j}$ for all $i, j=1,2, \ldots, n$.

Lemma 2.2 [11] (Generalized Hölder's inequality) Let $a_{r j}>0(j=1,2, \ldots, m, r=1,2, \ldots, n)$. If $\lambda_{1} \neq 0, \lambda_{j}<0(j=2,3, \ldots, m), \sum_{j=1}^{m} \frac{1}{\lambda_{j}} \leq 1$, then

$$
\sum_{r=1}^{n} \prod_{j=1}^{m} a_{r j} \geq \prod_{j=1}^{m}\left(\sum_{r=1}^{n} a_{r j}^{\lambda_{j}}\right)^{\frac{1}{\lambda_{j}}}
$$

The sign of the equality holds if and only if the $m$ sets $\left(a_{r 1}\right),\left(a_{r 2}\right), \ldots,\left(a_{r m}\right)$ are proportional. 
Lemma 2.3 Let $a_{r j}>0(r=1,2, \ldots, n, j=1,2, \ldots, m)$, let $\lambda_{1} \neq 0, \lambda_{j}<0(j=2,3, \ldots, m)$, and let $\tau=\max \left\{\sum_{j=1}^{m} \frac{1}{\lambda_{j}}, 1\right\}$. Then

$$
\sum_{r=1}^{n} \prod_{j=1}^{m} a_{r j} \geq n^{1-\tau} \prod_{j=1}^{m}\left(\sum_{r=1}^{n} a_{r j}^{\lambda_{j}}\right)^{\frac{1}{\lambda_{j}}} .
$$

The sign of the equality holds if and only if the $m$ sets $\left(a_{r 1}\right),\left(a_{r 2}\right), \ldots,\left(a_{r m}\right)$ are proportional for $\sum_{j=1}^{m} \frac{1}{\lambda_{j}} \leq 1$ or $a_{1 j}=a_{2 j}=\cdots=a_{n j}, j=1,2, \ldots, m$ for $\sum_{j=1}^{m} \frac{1}{\lambda_{j}}>1$.

Proof Case (I). When $\lambda_{1}<0$, then $\tau=1$. Obviously, inequality (7) is equivalent to inequality (6).

Case (II). When $\lambda_{1}>0$ with $\sum_{j=1}^{m} \frac{1}{\lambda_{j}} \geq 1$. Write $\sum_{j=1}^{m} \frac{1}{\lambda_{j}}=t(t \geq 1)$, which implies $\sum_{j=1}^{m} \frac{1}{t \lambda_{j}}=1$. By inequality (6), we have

$$
\begin{aligned}
\left(\sum_{r=1}^{n} \prod_{j=1}^{m} a_{r j}\right)^{2}= & \sum_{s=1}^{n}\left(\prod_{i=1}^{m} a_{s i}\right) \sum_{r=1}^{n} \prod_{j=1}^{m} a_{r j} \\
\geq & \sum_{s=1}^{n}\left(\prod_{i=1}^{m} a_{s i}\right)\left[\prod_{j=1}^{m}\left(\sum_{r=1}^{n} a_{r j}^{t \lambda_{j}}\right)^{\frac{1}{t \lambda_{j}}}\right] \\
= & \sum_{s=1}^{n}\left\{\left(a_{s 1}^{t \lambda_{1}} \sum_{r=1}^{n} a_{r 1}^{t \lambda_{1}}\right)^{\frac{1}{t \lambda_{1}}-\sum_{j=2}^{m} \frac{1}{\frac{1}{t \lambda_{j}}}} \times\left[\prod_{j=2}^{m}\left(a_{s 1}^{t \lambda_{1}} \sum_{r=1}^{n} a_{r j}^{t \lambda_{j}}\right)^{\frac{1}{t \lambda_{j}}}\right]\right. \\
& \left.\times\left[\prod_{j=2}^{m}\left(a_{s j}^{t \lambda_{j}} \sum_{r=1}^{n} a_{r 1}^{t \lambda_{1}}\right)^{\frac{1}{t \lambda_{j}}}\right]\right\} .
\end{aligned}
$$

Consequently, according to $\left(\frac{1}{t \lambda_{1}}-\sum_{j=2}^{m} \frac{1}{t \lambda_{j}}\right)+\frac{1}{t \lambda_{2}}+\frac{1}{t \lambda_{3}}+\cdots+\frac{1}{t \lambda_{m}}+\frac{1}{t \lambda_{2}}+\frac{1}{t \lambda_{3}}+\cdots+\frac{1}{t \lambda_{m}}=1$, by using inequality (6) on the right side of (8), we observe that

$$
\begin{aligned}
\left(\sum_{r=1}^{n} \prod_{j=1}^{m} a_{r j}\right)^{2} \geq & \left(\sum_{s=1}^{n} \sum_{r=1}^{n} a_{s 1}^{t \lambda_{1}} a_{r 1}^{t \lambda_{1}}\right)^{\frac{1}{t \lambda_{1}}-\sum_{j=2}^{m} \frac{1}{t \lambda_{j}}} \\
& \times\left[\prod_{j=2}^{m}\left(\sum_{s=1}^{n} \sum_{r=1}^{n} a_{s 1}^{t \lambda_{1}} a_{r j}^{t \lambda_{j}}\right)^{\frac{1}{t \lambda_{j}}}\right]\left[\prod_{j=2}^{m}\left(\sum_{s=1}^{n} \sum_{r=1}^{n} a_{s j}^{t \lambda_{j}} a_{r 1}^{t \lambda_{1}}\right)^{\frac{1}{t \lambda_{j}}}\right] .
\end{aligned}
$$

Additionally, using Lemma 2.1 together with $t \geq 1$, we find

$$
\begin{gathered}
\left(\sum_{s=1}^{n} \sum_{r=1}^{n} a_{s 1}^{t \lambda_{1}} a_{r 1}^{t \lambda_{1}}\right)^{\frac{1}{t \lambda_{1}}-\sum_{j=2}^{m} \frac{1}{t \lambda_{j}}} \times\left[\prod_{j=2}^{m}\left(\sum_{s=1}^{n} \sum_{r=1}^{n} a_{s 1}^{t \lambda_{1}} a_{r j}^{t \lambda_{j}}\right)^{\frac{1}{t \lambda_{j}}}\right] \\
\times\left[\prod_{j=2}^{m}\left(\sum_{s=1}^{n} \sum_{r=1}^{n} a_{s j}^{t \lambda_{j}} a_{r 1}^{t \lambda_{1}}\right)^{\frac{1}{t \lambda_{j}}}\right] \\
\geq\left(n^{2}\right)^{(1-t)\left(\frac{1}{t \lambda_{1}}-\sum_{j=2}^{m} \frac{1}{t \lambda_{j}}\right)}\left(\sum_{s=1}^{n} \sum_{r=1}^{n} a_{s 1}^{\lambda_{1}} a_{r 1}^{\lambda_{1}}\right)^{\frac{1}{\lambda_{1}}-\sum_{j=2}^{m} \frac{1}{\lambda_{j}}}
\end{gathered}
$$




$$
\begin{aligned}
& \times\left[\prod_{j=2}^{m}\left(n^{2}\right)^{\frac{1-t}{t \lambda_{j}}}\left(\sum_{s=1}^{n} \sum_{r=1}^{n} a_{s 1}^{\lambda_{1}} a_{r j}^{\lambda_{j}}\right)^{\frac{1}{\lambda_{j}}}\right]\left[\prod_{j=2}^{m}\left(n^{2}\right)^{\frac{1-t}{t_{j}}}\left(\sum_{s=1}^{n} \sum_{r=1}^{n} a_{s j}^{\lambda_{j}} a_{r 1}^{\lambda_{1}}\right)^{\frac{1}{\lambda_{j}}}\right] \\
&=\left(n^{2}\right)^{1-t}\left(\sum_{s=1}^{n} \sum_{r=1}^{n} a_{s 1}^{\lambda_{1}} a_{r 1}^{\lambda_{1}}\right)^{\frac{1}{\lambda_{1}}-\sum_{j=2}^{m} \frac{1}{\lambda_{j}}} \\
& \times\left[\prod_{j=2}^{m}\left(\sum_{s=1}^{n} \sum_{r=1}^{n} a_{s 1}^{\lambda_{1}} a_{r j}^{\lambda_{j}}\right)^{\frac{1}{\lambda_{j}}}\right]\left[\prod_{j=2}^{m}\left(\sum_{s=1}^{n} \sum_{r=1}^{n} a_{s j}^{\lambda_{j}} a_{r 1}^{\lambda_{1}}\right)^{\frac{1}{\lambda_{j}}}\right] \\
&= n^{2-2 t}\left(\sum_{r=1}^{n} a_{r 1}^{\lambda_{1}}\right)^{\frac{2}{\lambda_{1}}-\sum_{j=2}^{m} \frac{2}{\lambda_{j}}} \times\left\{\prod _ { j = 2 } ^ { m } \left[\left(\sum_{s=1}^{n} \sum_{r=1}^{n} a_{s 1}^{\lambda_{1}} a_{r j}^{\lambda_{j}}\right)\right.\right. \\
&\left.\left.\times\left(\sum_{s=1}^{n} \sum_{r=1}^{n} a_{s j}^{\lambda_{j}} a_{r 1}^{\lambda_{1}}\right)\right]^{\frac{1}{\lambda_{j}}}\right\} \\
&= n^{2-2 t}\left(\sum_{r=1}^{n} a_{r 1}^{\lambda_{1}}\right)^{\frac{2}{\lambda_{1}}-\sum_{j=2}^{m} \frac{2}{\lambda_{j}}} \times\left\{\prod _ { j = 2 } ^ { m } \left[\left(\sum_{s=1}^{n} a_{s 1}^{\lambda_{1}}\right)\left(\sum_{r=1}^{n} a_{r j}^{\lambda_{j}}\right)\right.\right. \\
&\left.\left.\times\left(\sum_{s=1}^{n} a_{s j}^{\lambda_{j}}\right)\left(\sum_{r=1}^{n} a_{r 1}^{\lambda_{1}}\right)\right]^{\frac{1}{\lambda_{j}}}\right\} \\
&= n^{2-2 t} \prod_{j=1}^{m}\left(\sum_{r=1}^{n} a_{r j}^{\lambda_{j}}\right)^{\frac{2}{\lambda_{j}}} \cdot \\
&
\end{aligned}
$$

Combining inequalities (9) and (10) leads to inequality (7) immediately.

Case (III). When $\lambda_{1}>0$ with $\sum_{j=1}^{m} \frac{1}{\lambda_{j}} \leq 1$. Obviously, inequality (7) is equivalent to inequality (6).

The condition of the equality for inequality can easily be obtained by Lemma 2.1 and Lemma 2.2. This completes the proof of Lemma 2.3.

Remark 2.4 It is clear that the generalized Hölder inequality (6) is a simple consequence of Lemma 2.3 presented in this article.

Theorem 2.5 Let $a_{r j}>0, \lambda_{1} \neq 0, \lambda_{j}<0(j=2,3, \ldots, m), a_{1 j}^{\lambda_{j}}-\sum_{r=2}^{n} a_{r j}^{\lambda_{j}}>0, r=1,2, \ldots, n$, $j=1,2, \ldots, m$, and let $\tau=\max \left\{\sum_{j=1}^{m} \frac{1}{\lambda_{j}}, 1\right\}$. Then

$$
\prod_{j=1}^{m}\left(a_{1 j}^{\lambda_{j}}-\sum_{r=2}^{n} a_{r j}^{\lambda_{j}}\right)^{\frac{1}{\lambda_{j}}} \geq n^{1-\tau} \prod_{j=1}^{m} a_{1 j}-\sum_{r=2}^{n} \prod_{j=1}^{m} a_{r j},
$$

and the equality holds if and only if $a_{1 j}=n^{\frac{1}{\lambda_{j}}} a_{2 j}=\cdots=n^{\frac{1}{\lambda_{j}}} a_{n j}, j=1,2, \ldots, m$ for $\tau>1$, or

$$
\frac{a_{11}^{\lambda_{1}}}{a_{1 j}^{\lambda_{j}}}=\frac{a_{21}^{\lambda_{1}}}{a_{2 j}^{\lambda_{j}}}=\cdots=\frac{a_{n 1}^{\lambda_{1}}}{a_{n j}^{\lambda_{j}}}, \quad j=2,3, \ldots, m \text { for } \tau=1 .
$$


Proof Denote

$$
a_{1 j}^{\lambda_{j}}-\sum_{r=2}^{n} a_{r j}^{\lambda_{j}}=x_{j}^{\lambda_{j}}
$$

and

$$
\prod_{j=1}^{m} a_{1 j}-n^{\tau-1} \sum_{r=2}^{n} \prod_{j=1}^{m} a_{r j}=n^{\tau-1} \prod_{j=1}^{m} x_{j}
$$

By using inequality (7), we have

$$
\prod_{j=1}^{m} a_{1 j}=\prod_{j=1}^{m}\left(x_{j}^{\lambda_{j}}+\sum_{r=2}^{n} a_{r j}^{\lambda_{j}}\right)^{\frac{1}{\lambda_{j}}} \leq n^{\tau-1}\left(\prod_{j=1}^{m} x_{j}+\sum_{r=2}^{n} \prod_{j=1}^{m} a_{r j}\right)
$$

that is,

$$
\left(x_{m}^{\lambda_{m}}+\sum_{r=2}^{n} a_{r m}^{\lambda_{m}}\right)^{\frac{1}{\lambda_{m}}} \prod_{j=1}^{m-1}\left(x_{j}^{\lambda_{j}}+\sum_{r=2}^{n} a_{r j}^{\lambda_{j}}\right)^{\frac{1}{\lambda_{j}}} \leq n^{\tau-1}\left(\prod_{j=1}^{m} x_{j}+\sum_{r=2}^{n} \prod_{j=1}^{m} a_{r j}\right) .
$$

Therefore, from (12), (13) and (15), we obtain

$$
\left(x_{m}^{\lambda_{m}}+\sum_{r=2}^{n} a_{r m}^{\lambda_{m}}\right)^{\frac{1}{\lambda_{m}}} \prod_{j=1}^{m-1} a_{1 j} \leq \prod_{j=1}^{m} a_{1 j}
$$

Hence, we obtain

$$
x_{m}^{\lambda_{m}} \geq a_{1 m}^{\lambda_{m}}-\sum_{r=2}^{m} a_{r m}^{\lambda_{m}}
$$

that is,

$$
x_{m} \leq\left(a_{1 m}^{\lambda_{m}}-\sum_{r=2}^{m} a_{r m}^{\lambda_{m}}\right)^{\frac{1}{\lambda_{m}}} .
$$

Therefore, we have

$$
\begin{aligned}
\prod_{j=1}^{m} x_{j} & \leq\left(a_{1 m}^{\lambda_{m}}-\sum_{r=2}^{m} a_{r m}^{\lambda_{m}}\right)^{\frac{1}{\lambda_{m}}} \prod_{j=1}^{m-1} x_{j} \\
& =\left(a_{1 m}^{\lambda_{m}}-\sum_{r=2}^{m} a_{r m}^{\lambda_{m}}\right)^{\frac{1}{\lambda_{m}}} \prod_{j=1}^{m-1}\left(a_{1 j}^{\lambda_{j}}-\sum_{r=2}^{m} a_{r j}^{\lambda_{j}}\right)^{\frac{1}{\lambda_{j}}} \\
& =\prod_{j=1}^{m}\left(a_{1 j}^{\lambda_{j}}-\sum_{r=2}^{m} a_{r j}^{\lambda_{j}}\right)^{\frac{1}{\lambda_{j}}} .
\end{aligned}
$$


By using (13), we immediately obtain the desired inequality (11). The condition of the equality for inequality (11) can easily be obtained by Lemma 2.3. The proof of Theorem 2.5 is completed.

If we set $\sum_{j=1}^{m} \frac{1}{\lambda_{j}} \leq 1$, then from Theorem 2.5 , we obtain the following reversed version of inequality (3).

Corollary 2.6 Let $a_{r j}>0, \lambda_{1} \neq 0, \lambda_{j}<0(j=2,3, \ldots, m), \sum_{j=1}^{m} \frac{1}{\lambda_{j}} \leq 1, a_{1 j}^{\lambda_{j}}-\sum_{r=2}^{n} a_{r j}^{\lambda_{j}}>0$, $r=1,2, \ldots, n, j=1,2, \ldots, m$. Then

$$
\prod_{j=1}^{m}\left(a_{1 j}^{\lambda_{j}}-\sum_{r=2}^{n} a_{r j}^{\lambda_{j}}\right)^{\frac{1}{\lambda_{j}}} \geq \prod_{j=1}^{m} a_{1 j}-\sum_{r=2}^{n} \prod_{j=1}^{m} a_{r j} .
$$

If we set $m=2, \lambda_{1}=p \neq 0, \lambda_{2}=q<0, a_{r 1}=a_{r}, a_{r 2}=b_{r}(r=1,2, \ldots, n)$, then from Theorem 2.5 , we obtain

Corollary 2.7 Let $a_{r}>0, b_{r}>0(r=1,2, \ldots, n), a_{1}^{p}-\sum_{r=2}^{n} a_{r}^{p}>0, b_{1}^{q}-\sum_{r=2}^{n} b_{r}^{q}>0, p \neq 0$, $q<0, \rho=\max \left\{\frac{1}{p}+\frac{1}{q}, 1\right\}$. Then the following inequality holds:

$$
\left(a_{1}^{p}-\sum_{r=2}^{n} a_{r}^{p}\right)^{\frac{1}{p}}\left(b_{1}^{q}-\sum_{r=2}^{n} b_{r}^{q}\right)^{\frac{1}{q}} \geq n^{1-\rho} a_{1} b_{1}-\sum_{r=2}^{n} a_{r} b_{r} .
$$

Remark 2.8 For $\frac{1}{p}+\frac{1}{q}=1$, inequality (21) reduces to the famous Aczél-Vasić-Pečarić inequality (2).

\section{Application}

As application of the above results, we establish here an integral type of the reversed version of the Aczél-Vasić-Pečarić inequality.

Theorem 3.1 Let $\lambda_{1}>0, \lambda_{j}<0(j=2,3, \ldots, m), \sum_{j=1}^{m} \lambda_{j}=1$, let $A_{j}>0(j=1,2, \ldots, m)$, and let $f_{j}(x)(j=1,2, \ldots, m)$ be positive Riemann integrable functions on $[a, b]$ such that $A_{j}^{\lambda_{j}}-$ $\int_{a}^{b} f_{j}^{\lambda_{j}}(x) \mathrm{d} x>0$. Then

$$
\prod_{j=1}^{m}\left(A_{j}^{\lambda_{j}}-\int_{a}^{b} f_{j}^{\lambda_{j}}(x) \mathrm{d} x\right)^{\frac{1}{\lambda_{j}}} \geq \prod_{j=1}^{m} A_{j}-\int_{a}^{b} \prod_{j=1}^{m} f_{j}(x) \mathrm{d} x .
$$

Proof For any positive integer $n$, we choose an equidistant partition of $[a, b]$ as

$$
\begin{aligned}
& a<a+\frac{b-a}{n}<\cdots<a+\frac{b-a}{n} k<\cdots<a+\frac{b-a}{n}(n-1)<b, \\
& x_{k}=a+\frac{b-a}{n} k, \quad \Delta x_{k}=\frac{b-a}{n}, \quad k=1,2, \ldots, n .
\end{aligned}
$$

Since the hypothesis $A_{j}^{\lambda_{j}}-\int_{a}^{b} f_{j}^{\lambda_{j}}(x) \mathrm{d} x>0(j=1,2, \ldots, m)$ implies that

$$
A_{j}^{\lambda_{j}}-\lim _{n \rightarrow \infty} \sum_{k=1}^{n} f_{j}^{\lambda_{j}}\left(a+\frac{k(b-a)}{n}\right) \frac{b-a}{n}>0 \quad(j=1,2, \ldots, m),
$$


there exists a positive integer $N$ such that

$$
A_{j}^{\lambda_{j}}-\sum_{k=1}^{n} f_{j}^{\lambda_{j}}\left(a+\frac{k(b-a)}{n}\right) \frac{b-a}{n}>0 \text { for all } n>N \text { and } j=1,2, \ldots, m \text {. }
$$

By using Theorem 2.5, we obtain that for any $n>N$, the following inequality holds:

$$
\begin{aligned}
& \prod_{j=1}^{m}\left[A_{j}^{\lambda_{j}}-\sum_{k=1}^{n} f_{j}^{\lambda_{j}}\left(a+\frac{k(b-a)}{n}\right) \frac{b-a}{n}\right]^{\frac{1}{\lambda_{j}}} \\
& \geq \prod_{j=1}^{m} A_{j}-\sum_{k=1}^{n}\left[\prod_{j=1}^{m} f_{j}\left(a+\frac{k(b-a)}{n}\right)\right]\left(\frac{b-a}{n}\right)^{\sum_{j=1}^{m} \frac{1}{\lambda_{j}}} .
\end{aligned}
$$

Since

$$
\sum_{j=1}^{m} \frac{1}{\lambda_{j}}=1
$$

we have

$$
\begin{aligned}
& \prod_{j=1}^{m}\left[A_{j}^{\lambda_{j}}-\sum_{k=1}^{n} f_{j}^{\lambda_{j}}\left(a+\frac{k(b-a)}{n}\right) \frac{b-a}{n}\right]^{\frac{1}{\lambda_{j}}} \\
& \geq \prod_{j=1}^{m} A_{j}-\sum_{k=1}^{n}\left[\prod_{j=1}^{m} f_{j}\left(a+\frac{k(b-a)}{n}\right)\right]\left(\frac{b-a}{n}\right) .
\end{aligned}
$$

In view of the hypotheses that $f_{j}(x)(j=1,2, \ldots, m)$ are positive Riemann integrable functions on $[a, b]$, we conclude that $\prod_{j=1}^{m} f_{j}(x)$ and $f_{j}^{\lambda_{j}}(x)$ are also integrable on $[a, b]$. Passing the limit as $n \rightarrow \infty$ on both sides of inequality (24), we obtain inequality (22). The proof of Theorem 3.1 is completed.

\section{Acknowledgements}

The author would like to express his sincere thanks to the anonymous referees for their great efforts to improve this paper. This work was supported by the NNSF of China (Grant No. 61073121), and the Fundamental Research Funds for the Central Universities (No. 11ML65)

\section{Received: 3 February 2012 Accepted: 21 August 2012 Published: 11 September 2012}

\section{References}

1. Aczél, J: Some general methods in the theory of functional equations in one variable, new applications of functional equations. Usp. Mat. Nauk 11(3), 3-68 (1956) (in Russian)

2. Beckenbach, EF, Bellman, R: Inequalities. Springer, Berlin (1983)

3. Díaz-Barrerro, JL, Grau-Sánchez, M, Popescu, PG: Refinements of Aczél, Popoviciu and Bellman's inequalities. Comput. Math. Appl. 56, 2356-2359 (2008)

4. Farid, G, Pečarić, J, Ur Rehman, A: On refinements of Aczél's, Popoviciu, Bellman's inequalities and related results. J. Inequal. Appl. 2010, 579567 (2010)

5. Hardy, G, Littlewood, JE, Pólya, G: Inequalities. Cambridge University Press, UK (1952)

6. Mitrinović, DS, Pečarić, JE, Fink, AM: Classical and New Inequalities in Analysis. Kluwer Academic, Dordrecht (1993)

7. Ouyang, Y, Mesiar, R: On the Chebyshev type inequality for seminormed fuzzy integral. Appl. Math. Lett. 22(12), 1810-1815 (2009)

8. Popoviciu, T: On an inequality. Gaz. Mat. Fiz., Ser. A 11(64), 451-461 (1959) (in Romanian)

9. Tian, J: Inequalities and mathematical properties of uncertain variables. Fuzzy Optim. Decis. Mak. 10(4), 357-368 (2011) 
10. Vasić, PM, Pečarić, JE: On Hölder and some related inequalities. Mathematica Rev. D'Anal. Num. Th. L’Approx. 25, 95-103 (1982)

11. Vasić, PM, Pečarić, JE: On the Jensen inequality for monotone functions. An. Univ. Timişoara Ser. Şt. Matematice 17(1), 95-104 (1979)

12. Vong, S: On a generalization of Aczél's inequality. Appl. Math. Lett. 24, 1301-1307 (2011)

13. Yang, W: Refinements of generalized Aczél-Popoviciu's inequality and Bellman's inequality. Comput. Math. Appl. 59, 3570-3577 (2010)

14. Wu, S, Debnath, L: Generalizations of Aczél's inequality and Popoviciu's inequality. Indian J. Pure Appl. Math. 36(2), 49-62 (2005)

doi:10.1186/1029-242X-2012-202

Cite this article as: Tian: Reversed version of a generalized Aczél's inequality and its application. Journal of Inequalities and Applications 2012 2012:202.

Submit your manuscript to a SpringerOpen ${ }^{\odot}$ journal and benefit from:

- Convenient online submission

- Rigorous peer review

- Immediate publication on acceptance

- Open access: articles freely available online

- High visibility within the field

- Retaining the copyright to your article

Submit your next manuscript at $>$ springeropen.com 